Referencia para citar este artículo: Forero, I. Siabato, E. \& Salamanca, Y. (2017). Ideación suicida, funcionalidad familiar y consumo de alcohol en adolescentes de Colombia. Revista Latinoamericana de Ciencias Sociales, Niñez y Juventud, 15(1), pp. 431-442.

\title{
Ideación suicida, funcionalidad familiar y consumo de alcohol en adolescentes de Colombia*
}

\author{
INGRID FORERo** \\ Profesora-Investigadora Universidad Pedagógica y Tecnológica, Colombia. \\ ELSA SIABATO**** \\ Investigadora Universidad Pedagógica y Tecnológica, Colombia. \\ YENNY $S_{A L A M A N C A}^{* * * *}$ \\ Profesora Universidad Pedagógica y Tecnológica, Colombia.
}

\section{Artículo recibido en abril 29 de 2016; artículo aceptado en julio 18 de 2016 (Eds.)}

- Resumen (analítico): Estudio no experimental, de tipo descriptivo, cuyo objetivo fue analizar la asociación entre ideación suicida, funcionalidad familiar y consumo de alcohol en 289 adolescentes (126 hombres y 163 mujeres) estudiantes de colegios públicos de Tunja - Colombia, con edades comprendidas entre los 13 y los 17 años, efectuado mediante el Inventario de Ideación Suicida Positiva y Negativa (Pansi), el cuestionario Apgar familiar, y el cuestionario de identificación de trastornos debidos al consumo de alcohol (Audit). Los resultados indican que el 30\% de los individuos participantes refirieron un nivel alto de ideación suicida, el 67\% un buen funcionamiento familiar, y el 84\% consumo de alcohol de bajo riesgo; así mismo, identificamos una asociación mayor entre ideación suicida y el tipo de funcionalidad familiar grave.

Palabras clave: Adolescentes, alcoholismo, relaciones familiares, ideación suicida, psicología (Tesauro DeCS-Descriptores en Ciencias de la Salud).

\section{Suicidal ideation, family functioning and alcohol consumption among Colombian adolescents}

- Abstract (analytical): This article describes a non-experimental descriptive study with the objective of analyzing the relationship between suicidal ideation, family functioning and consumption of alcohol among 289 adolescents (126 men and 163 women) who are students from public schools in Tunja-Colombia aged between 13 and 17 years. The authors carried out this analysis using the

\footnotetext{
* Este artículo es de investigación científica y tecnológica. Área Psicología, subárea Psicología. Presenta los resultados del proyecto denominado "Factores psicosociales asociados a ideación suicida en estudiantes de colegios públicos de Tunja" SGI 1520 del grupo de investigación clínica y salud, avalado por la Universidad Pedagógica y Tecnológica de Colombia en el marco del Programa de Jóvenes Investigadores e Innovadores de Colciencias. Inicio: Abril de 2014-Finalización: Mayo de 2015.

** Psicóloga UPTC. Mg. (c) en Educación UPTC. Joven Investigadora Colciencias-UPTC Grupo de Investigación Clínica y Salud. Correo electrónico: elsa.siabato@uptc.edu.co

*** Psicóloga UPTC. Especialista en necesidades de aprendizaje lectura, escritura y matemáticas. Mg. (c) Psicología con énfasis en Clínica Universidad del Norte. Joven Investigadora Colciencias-UPTC Grupo de Investigación Clínica y Salud. Correo electrónico: elsa.siabato@uptc. edu.co

**** Psicóloga UPTC. Ms.C. en Psicología Clínica y de la Salud; Mg. En Psicología, Universidad del Norte; Ph. D. (c) Psicología Clínica y de la Salud UGR; docente Escuela de Psicología y coordinadora Grupo de Investigación Clínica y Salud, UPTC. Correo electrónico: yenny.salamanca@uptc. edu.co
} 
following tools: Inventory of Positive and Negative Suicidal Ideation (Pansi), the family Apgar questionnaire and the Alcohol Use Disorders Identification Test (Audit). The results indicate that 30 $\%$ of participants reported a high level of suicidal ideation, 67\% reported that their family functions well and $84 \%$ demonstrated low-risk alcohol consumption. The study identified a strong relationship between suicidal ideation and serious family dysfunction.

Key words: Adolescents, alcoholism, family, suicidal ideation, psychology (DeCS-Health Sciences Descriptors Thesaurus).

\section{Ideação suicida, funcionamento familiar e consumo de álcool em adolescentes colombianos}

- $\quad$ Resumo (analítico): Estudo não experimental, descritivo, cujo objetivo foi analisar a associação entre ideação suicida, funcionamento familiar e consumo de álcool em 289 adolescentes (126 homens e 163 mulheres) estudantes de escolas públicas de Tunja (Colômbia) com idade entre 13 e 17 anos; a partir do Inventário de Ideação suicida Positiva e Negativa (Pansi), o questionário Apgar de família e o teste de identificação de desordens devido ao Álcool (Audit). Os resultados indicam que 30\% dos participantes relataram um alto nível de ideação suicida, 67\% um bom funcionamento familiar e 84\% um consumo de álcool de baixo risco; do mesmo modo, foi identificada uma associação mais forte entre ideação suicida e o tipo de funcionalidade familiar grave.

Palvras-chave: Adolescentes, alcoolismo, família, ideação suicida, psicologia(DeCS-Descritores em Ciências da Saúde Tesauro).

\section{-1. Introducción. -2. Método. -3. Resultados. -4. Discusión. -5. Conclusiones. -Lista de referencias.}

\section{Introducción}

Según la Organización Mundial de la Salud (OMS, 2014), dentro de las muertes violentas los suicidios representan un $50 \%$ en hombres y un $71 \%$ en mujeres, siendo la segunda causa principal de muerte en personas con edades comprendidas entre los 15 y los 29 años. En Colombia, es considerada la cuarta forma de violencia, encontrándose que por cada mujer, 4 hombres se quitan la vida, siendo mayor el número de casos registrados entre los 20 y $\operatorname{los} 24$ años de edad (Instituto Nacional de Medicina Legal y Ciencias ForensesINMLCF, 2013); sumado a estas estadísticas, es importante tener en cuenta lo ya reportado en el informe de salud de la OMS (2014) con respecto a que el problema podría ser más grave de lo que parece, ya que se pretende esconder la situación con el fin de evitar la señalización de la familia o de la persona, ya sea por beneficio social, pensamientos políticos o porque quien se suicida lo hace parecer como un accidente (Sánchez, Villareal, Musitu \& Martínez, 2010).

Al respecto, Pérez, Téllez, Vélez e Ibáñez (2012), refieren que las tasas de suicidio en adolescentes de Colombia, han venido en aumento en los últimos años, y que aunque las cifras de suicidio consumado no son tan elevadas, los datos de intento e ideación suicida hacen que el fenómeno sea preocupante; razón por la cual, el estudiar los determinantes del suicidio como estrategia de prevención, se convierte en un tema de especial interés no solamente para los profesionales de la salud y la educación, sino para los propios individuos y sus familias. Investigaciones relacionadas indican la importancia de estudiar variables tales como la familia, ya que como mencionan García y Calíope (1998), el aumento en la cohesión familiar se convierte en un factor de protección ante el intento suicida; así mismo, De Wilde (2000) reconoce la influencia que ejerce el grupo familiar sobre dicha conducta, y Cabra, Infante y Sossa (2010) resaltan el rol que tiene la familia y las relaciones familiares como factor de riesgo o protección en la conducta de los sujetos adolescentes.

En cuanto al consumo de alcohol, Bailador, Viscardi y Dajas (1997) realizaron un estudio en Montevideo (Uruguay), en el que encontraron una asociación significativa 
entre depresión, ideación suicida, consumo de alcohol y percepción de conflictos en la familia; así mismo, Evans, Hawton y Rodhan (2004), y Gómez, Rodríguez, Bohórquez, Diazgranados, Ospina y Fernández (2002), encontraron una asociación entre la ingesta de bebidas con alto contenido de licor y la ideación suicida; y finalmente, Ramírez y Andrade (2005) encontraron una relación entre el consumo de alcohol y el ambiente y funcionamiento familiar en el que se involucran aspectos tales como capacidad de adaptación, grado de participación, afectividad, autorrealización gracias al apoyo familiar y compromiso entre los miembros de la familia.

Al analizar la prevalencia de consumo, Camacho (2005) refiere un 70\% para estudiantes de secundaria; mientras que el estudio nacional sobre consumo de sustancias psicoactivas en escolares (Ministerio de Justicia de Colombia, Ministerio de Salud de Colombia \& Observatorio de Drogas de Colombia, 2013), refiere a que del total de estudiantes con edades entre $11 \mathrm{y}$ 18 años, el 40\% indicó haber consumido algún tipo de bebida alcohólica durante el último mes; con respecto al consumo según el género, las cifras no son muy distantes, ya que el $39.5 \%$ de mujeres revelaron haber consumido alcohol respecto al $40.1 \%$ de hombres; cabe resaltar que en este informe también se mencionan los conflictos familiares como una de las justificaciones para el suicidio, soportando así los planteamientos de Campo et al. (2003), quienes ya habían afirmado que la carencia de apoyo familiar y la disfunción familiar severa interviene de manera significativa en la salud mental del individuo adolescente y por ende está relaciona con el suicidio.

Por su parte, la OMS (2014), en su informe sobre consumo de alcohol, indica que el inicio antes de los 14 años de edad puede predecir un estado de salud perjudicial; de igual manera, esta misma organización (OMS, 2015) advierte que "las enfermedades mentales, especialmente la depresión y los trastornos por consumo de alcohol, abuso de sustancias, violencia, sensaciones de pérdida y los distintos entornos sociales y culturales constituyen factores de riesgo para el suicidio".

Rev.latinoam.cienc.soc.niñez juv 15 (1): 431-442, 2017 http://revistalatinoamericanaumanizales.cinde.org.co DOI:10.11600/1692715x.1512729042016
Teniendo en cuenta los estudios realizados en Colombia sobre ideación suicida, se resalta el de Valencia en 2014, quien analizó esta variable en 424 estudiantes de una institución educativa de Chiquinquirá, y encontró que el $29 \%$ de los participantes reportaron un nivel alto $\mathrm{y}$ muy alto de ideación suicida; por su parte, al analizar los factores de riesgo para la conducta suicida, se encuentra que variables tales como el consumo de sustancias psicoactivas y las relaciones familiares pobres, entre otras variables relacionadas con la salud mental y la calidad de vida, son determinantes para dicha conducta (Fuentes et al., 2009, Pérez et al., 2012, y Paniagua, González \& Rueda, 2014).

Al respecto, Pérez et al. (2012), pretendieron caracterizar los factores asociados con comportamiento suicida en estudiantes de grado octavo, y concluyeron que el riesgo de conducta suicida fue explicado entre otras por la disfunción familiar severa $(\mathrm{OR}=3,4, \mathrm{IC} 95 \%$ $=1,2-9,7)$; de la misma manera, Paniagua et al. (2014), en una investigación realizada en Medellín con 604 adolescentes escolarizados, determinaron que esta variable es la que mejor explica el riesgo de orientación suicida $(\mathrm{OR}=$ 2,22; IC 95\% 1,44-3,42); sin embargo, Pérez, Vianchá, Martínez y Salas (2014), encontraron una correlación débil entre las dos variables $(0,173)$ presentando el $5,6 \%$ de la muestra ideación suicida.

Específicamente, en cuanto al consumo de alcohol, la funcionalidad familiar y la ideación suicida, Fuentes et al. (2009) realizaron un estudio con adolescentes de educación media, y encontraron que el 39,2\% son dependientes del alcohol, y que existe una relación significativa entre el consumo de alcohol, la disfunción familiar severa $(11,4 \%)$ y el riesgo suicida en dicha población; al respecto, es de resaltar como factor de riesgo que Boyacá, según el Estudio Nacional de Consumo de Sustancias Psicoactivas en Colombia, ENCSPE, reveló que es uno de los departamentos con más altos niveles de consumo de alcohol.

Teniendo en cuenta los anteriores hallazgos, con el presente estudio pretendemos describir y analizar la asociación entre ideación suicida, funcionalidad familiar y consumo de alcohol en adolescentes de colegios públicos de la ciudad de Tunja. 


\section{Método}

\section{Tipo de investigación}

Se trata de un estudio cuantitativo con un diseño no experimental transeccional descriptivo, donde se observan situaciones existentes y que no son provocadas de manera intencional; los datos se recogen en un momento especifico (Hernández, Fernández \& Baptista, 2000).

\section{Participantes}

289 participantes (126 hombres y 163 mujeres), con edades comprendidas entre los 13 y los 17 años, y escolaridad entre noveno y once grado, de cuatro instituciones educativas públicas de la ciudad de Tunja. Realizamos la selección de la muestra a partir de la técnica de muestreo, por cuota porcentual, por género y grado, con una probabilidad a favor de .25 y un error de estimación del 5\%.

\section{Instrumentos}

Ideación suicida. Evaluada a partir del Inventario de ideación suicida positiva y negativa (Pansi) desarrollado por Osman, Gutiérrez, Jiandani, Barrios, Linden y Truelove (2003), y validado para Colombia por Villalobos-Galvis (2009a), en una muestra de estudiantes de colegios y universidades; presentando buena fiabilidad (.89). Es un cuestionario tipo Likert de 5 puntos que oscilan entre 0 (nunca) y 4 (siempre); cuenta con 14 ítems que evalúan lo sucedido en las últimas dos semanas y en los que se pregunta por la frecuencia en la presentación de cada uno de los 14 pensamientos, 6 de ideación suicida positiva que se refiere a los factores protectores, y 8 de ideación suicida negativa (factores de riesgo); al final obtenemos una puntuación total que indica la presencia de ideación suicida total.

Funcionalidad Familiar. Para la medición de ésta hicimos uso del Apgar Familiar, instrumento desarrollado por Smilkstein (1978); se trata de un cuestionario de 5 preguntas que explora el impacto de la función familiar sobre el comportamiento y la salud del individuo, a partir de cinco funciones básicas de la familia: adaptación, participación, gradiente de recurso personal, afecto, y recurso
(Suárez \& Alcalá, 2014). Este instrumento se encuentra validado en la población colombiana por Forero, Avendaño, Duarte y Campo (2006); presentó un alfa de Cronbach de 0.793 y el análisis factorial mostró que la escala estaba compuesta por un único factor que explicaba el 55,6\% de la varianza. Los ítems de la escala se califican como nunca, casi nunca, algunas veces, casi siempre y siempre, y se les asignan puntuaciones de 0 a 4 respectivamente. En este instrumento, los puntajes totales pueden oscilar entre 0 y 20; a mayor puntaje, mejor funcionalidad familiar. Una puntuación total entre 0 y 9 indica disfunción grave; entre 10 y 13 moderada; entre 14 y 17 leve, y si es igual o superior a 18 se considera funcional.

Consumo de Alcohol. Medido a través del Alcohol Use Disorders Identification Test (Audit), el cual se basa en un proyecto de la OMS, estandarizado posteriormente por Saunders, Aasland, Babor, De La Fuente y Grant (1993). Se trata de un cuestionario autoadministrado de 10 preguntas; las primeras 3 preguntas se refieren a cantidad y frecuencia del consumo de alcohol, las siguientes 3 se refieren a la actitud frente a la bebida, las preguntas 7 y 8 se relacionan con reacciones adversas y las últimas 2 preguntas están relacionadas con problemas debidos al consumo. Las primeras 8 preguntas tienen 5 opciones de respuesta con puntuaciones de 0 a 4 , mientras que las dos últimas sólo cuentan con 3 opciones $(0,2$, 4). La puntuación total del cuestionario oscila entre 0 y 40 . Este instrumento cuenta con un alfa de cronbach de 0.8 .

El instrumento permite identificar la presencia de un consumo de riesgo, consumo perjudicial o dependencia de alcohol; entendiéndose el consumo de riesgo como un patrón de consumo que aumenta la probabilidad de consecuencias adversas para el individuo consumidor o para los demás, en tanto el consumo perjudicial se refiere a aquel que conlleva consecuencias para la salud física y mental, así como consecuencias sociales; y finalmente la dependencia, que consiste en un conjunto de fenómenos conductuales, cognitivos y fisiológicos que pueden aparecer después del consumo repetido de alcohol, que incluye el deseo intenso de consumir alcohol, dificultad 
para controlar el consumo, persistencia en éste a pesar de las consecuencias perjudiciales, mayor prelación al consumo en contraste con otras actividades y obligaciones, aumento de la tolerancia al alcohol y abstinencia física al interrumpirse el consumo (OMS, 2001).

\section{Procedimiento}

En primer lugar seleccionamos las instituciones educativas en las cuales se llevaría a cabo el estudio, y solicitamos la respectiva autorización para realizar la investigación; posteriormente contactamos a los padres y madres de los sujetos participantes y se les explicó la naturaleza del estudio; una vez obtenido el consentimiento informado para participar en la investigación, procedimos al establecimiento del asentimiento informado y a la aplicación de los cuestionarios por pequeños grupos e institución educativa en una única sesión de 20 minutos por grupo.

\section{Análisis estadístico}

Con variables de estudio, realizamos un análisis descriptivo univariado y un análisis de normalidad mediante la prueba de Kolmogorov-Smirnov; así mismo, realizamos el análisis bivariado con el objetivo de analizar la asociación entre las variables ideación suicida, funcionalidad familiar y consumo de alcohol, utilizando la prueba Kruskal Wallis. El análisis estadístico lo realizamos con el paquete estadístico SPSS versión 22.0.

\section{Consideraciones Éticas}

Este estudio lo sustentamos en los principios establecidos en la ley 1090 de 2006 y en la resolución 008430 de 1993, las cuales regulan el ejercicio de la profesión psicológica en Colombia, así como la investigación en el mismo campo; para la aplicación de los cuestionarios psicológicos tuvimos en cuenta el capítulo VI del código; asimismo, los datos recolectados los manejamos con total confidencialidad, manteniendo siempre el anonimato de las personas participantes.

\section{Resultados}

Según el inventario Pansi, el 28,4\% presenta un nivel alto de ideación suicida negativa (ISN), el 28\% ideación suicida positiva (ISP), y en general, el $30 \%$ se ubica en un nivel alto de ideación suicida (IS); de este último porcentaje, en proporción el 36,8\% corresponde a las mujeres y el $20 \%$ a los hombres (Tabla, 1 ).

Tabla 1. Nivel de ideación suicida.

\begin{tabular}{|c|c|c|c|}
\hline Ideación Suicida & Nivel & Frecuencia & Porcentaje \\
\hline \multirow{3}{*}{ Positiva } & Bajo & 137 & 47,4 \\
\hline & Medio & 71 & 25,6 \\
\hline & Alto & 81 & 28 \\
\hline \multirow{3}{*}{ Negativa } & Bajo & 183 & 63,3 \\
\hline & Medio & 24 & 8,3 \\
\hline & Alto & 82 & 28,4 \\
\hline \multirow{3}{*}{ Total } & Bajo & 154 & 53,3 \\
\hline & Medio & 49 & 17 \\
\hline & Alto & 86 & 30 \\
\hline
\end{tabular}

Con respecto a los resultados arrojados a partir del cuestionario de funcionalidad familiar (Apgar), el 67\% de los estudiantes de la muestra perciben un buen funcionamiento de su familia, identificando que se permite el desarrollo de sus integrantes y se enfrentan adecuadamente a los problemas relacionales y de crisis familiar (Figura 1). 
Figura1. Distribución por Funcionalidad Familiar.

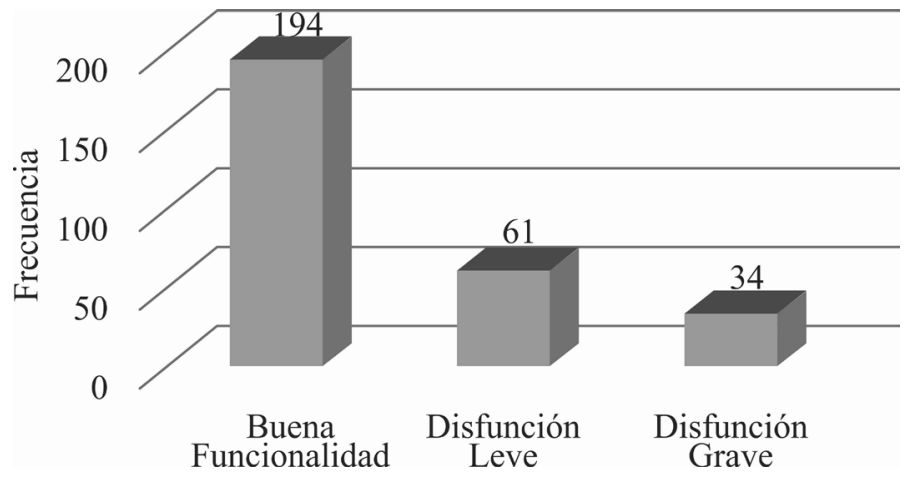

En cuanto al consumo de alcohol de acuerdo con los resultados obtenidos en el test Audit, la mayoría de los participantes reportaron un consumo moderado de alcohol,

mientras el $14 \%$ se encontraron en un consumo de riesgo que puede contraer consecuencias adversas tanto para sí mismos como para los demás (Figura, 2).

Figura 2. Distribución por Consumo de Alcohol.

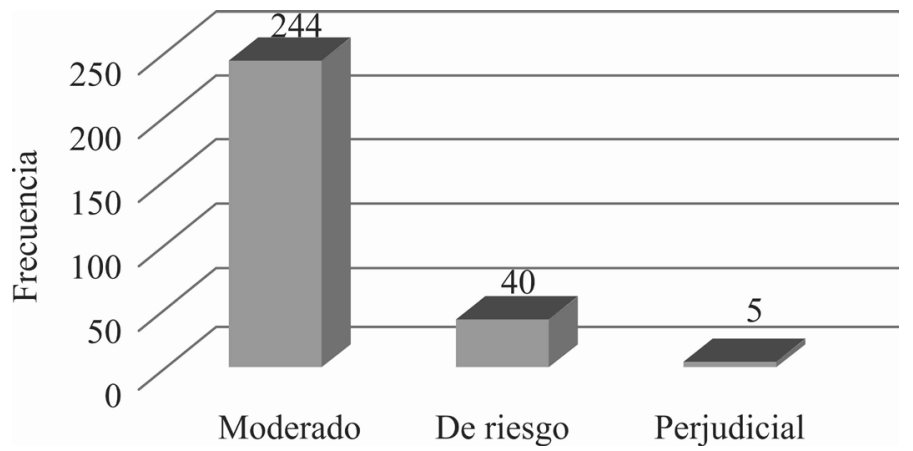

A continuación mostramos los resultados del análisis bivariado de las variables funcionalidad familiar y alcohol, respecto a ideación suicida:

En cuanto a la funcionalidad familiar, identificamos un mayor nivel de IS $\left(\chi^{2}=44,1\right.$ $p<.001)$ como de ISP $\left(\chi^{2}=30,1 p<.001\right)$ en quienes perciben disfuncionalidad grave. En relación a ISN no encontramos diferencias significativas (Tabla 2 ).

Tabla 2. Funcionalidad Familiar e Ideación suicida.

\begin{tabular}{cccccccc}
\hline $\begin{array}{c}\text { Fun. } \\
\text { Familiar }\end{array}$ & N & \multicolumn{2}{c}{ ISP } & \multicolumn{2}{c}{ ISN } & \multicolumn{2}{c}{ IS } \\
\hline & & Mdn & $\overline{\mathrm{R}}$ & Mdn & $\overline{\mathrm{R}}$ & Mdn & $\overline{\mathrm{R}}$ \\
\hline FF & 194 & 5 & 128,1 & 0 & 126,7 & 6 & 123,9 \\
\hline DL & 61 & 7 & 164,2 & 2 & 171,2 & 9 & 171,8 \\
\hline DG & 34 & 9,5 & 207 & 3,5 & 202,5 & 15 & 217,2 \\
\hline
\end{tabular}

Nota: FF=Funcionalidad Familiar, DL= Disfuncionalidad Leve, DG=Disfuncionalidad Grave, ISP=Ideación Suicida Positiva, ISN=Ideación Suicida Negativa, IS=Ideación Suicida. 
Respecto al consumo de alcohol y la ideación suicida no identificamos diferencias significativas (Tabla 3).

Tabla 3. Consumo de Alcohol e Ideación suicida.

\begin{tabular}{cccccccc}
\hline $\begin{array}{c}\text { Consumo } \\
\text { Alcohol }\end{array}$ & \multirow{2}{*}{$\mathrm{N}$} & \multicolumn{2}{c}{ ISP } & \multicolumn{2}{c}{ ISN } & \multicolumn{2}{c}{ IS } \\
\hline & & Mdn & $\overline{\mathrm{R}}$ & $\mathrm{Mdn}$ & $\overline{\mathrm{R}}$ & $\mathrm{Mdn}$ & $\overline{\mathrm{R}}$ \\
\hline Moderado & 244 & 5,5 & 140,5 & 0 & 144,3 & 7 & 141,8 \\
\hline De Riesgo & 40 & 6,5 & 166,6 & 0 & 146,2 & 7,5 & 160,1 \\
\hline Perjudicial & 5 & 8 & 189,5 & 1 & 163,3 & 9 & 182,7 \\
\hline
\end{tabular}

Nota: ISP= Ideación Suicida Positiva, ISN= Ideación Suicida Negativa, IS=Ideación Suicida.

\section{Discusión}

En general los hallazgos de este estudio nos permitieron conocer la situación actual de estudiantes de educación media de la ciudad de Tunja respecto a la ideación suicida, el funcionamiento familiar $\mathrm{y}$ el consumo de alcohol.

Con respecto a la ideación suicida, identificamos que el $30 \%$ de los sujetos participantes reportaron ideación suicida, de los cuales el $37 \%$ son mujeres y el $20 \%$ hombres; por su parte, Ceballos, Suarez, Suescún, Gamarra, González y Sotelo (2015), en el estudio realizado con adolescentes de Santa Marta, encontraron que el 13\% de las participantes presentan ideación suicida en riesgo medio y alto, y de los hombres el 23\% presenta ideación en nivel medio, asociándose la ideación con baja autoestima; entre tanto, Pérez, Rivera, Atienzo, Castro, Leyva y Chávez (2010) reportaron que el 47\% indicó al menos un síntoma de ideación, y un porcentaje menor refirió antecedente de intento; de manera tal que estas cifras parecen indicar la alta prevalencia de ideación suicida en estudiantes tanto en estudios previos como en éste; sin embargo, los resultados no son concluyentes en relación con que dependiendo del género aumente la presencia de conductas suicidas, tal como ya lo habían afirmado Ceballos et al. (2015), quienes tampoco hallaron relación entre el género y la ideación suicida.

Adicionalmente, cabe resaltar que diversos estudios han reportado la importancia de analizar este tema en adolescentes, ya que a partir de dichos resultados se puede estar alerta frente a los riesgos potenciales de ideación suicida, e iniciar los procedimientos de control a los que haya lugar (Córdova, Rosales, Guzmán \& Zúñiga, 2013), incluyendo el monitoreo constante mediante instrumentos de medición que posteriormente funcionan como ayudas diagnósticas para prevenir situaciones de riesgo (Paniagua et al., 2014), así como proponer estrategias de prevención y promoción en factores protectores en los que se resalte el papel tan importante que desempeña la familia, la escuela y el contexto sociocultural del adolescente (Pérez et al., 2010, Ceballos et al., 2015).

En cuanto a la variable función familiar, si bien el $67 \%$ de la población objeto de estudio presentó una buena funcionalidad familiar, es importante resaltar que el $12 \%$ de los individuos participantes refieren disfuncionalidad familiar grave, y a su vez reportan ideación suicida e ideación suicida positiva, lo cual, según Villalobos-Galvis (2009b), está relacionado con baja presencia de pensamientos de protección, tales como satisfacción con la vida y sentido de control y expectativas positivas; estos resultados son similares a los ya reportados por Fuentes et al. (2009) en su estudio realizado con 339 adolescentes, en donde encontraron que el $11.4 \%$ presentó disfuncionalidad severa; a su vez, soportan los planteamientos de Carvajal y Caro (2011) -quienes ya habían afirmado que características familiares como apoyo, adaptabilidad, cohesión y grado de disfunción, predicen tanto la ideación como el intento suicida-y de Cabra et al. (2010), quienes hacen referencia al aumento de la cohesión familiar como un factor protector de la conducta suicida, 
resaltando que la calidad en la interacción entre los sujetos integrantes y la convivencia familiar, es más importante que la estructura familiar en sí; aunque es de resaltar que al respecto Vargas y Saavedra (2012), reportaron que los resultados no parecen ser muy concluyentes, ya que a pesar de encontrarse una asociación significativa, la relación que se establece entre cohabitar con el padre y la madre y la conducta suicida, es indirecta.

Así mismo, se confirma lo mencionado en otras fuentes sobre la relación existente entre funcionalidad familiar y conducta suicida, en donde se ha planteado que los adolescentes y las adolescentes que perciben disfuncionalidad familiar grave son más propensos a presentar ideación suicida (Fuentes et al., 2009, Carvajal \& Caro, 2011, Cabra et al., 2010 y Vargas \& Saavedra, 2012).

Respecto a la variable consumo de alcohol, la mayoría de la población (84\%) reportó un consumo moderado, por lo que a pesar de que no se trate de un consumo de alto riesgo sí implica que los estudiantes y las estudiantes, a su corta edad, ya se encuentran inmersos en el consumo; al respecto, el Ministerio de Protección Social (MPS, 2005) manifestó su preocupación en relación con el aumento significativo de consumo de alcohol a temprana edad, y señaló que la ingesta de alcohol en los sujetos adolescentes se ha convertido en una de las mayores preocupaciones sociales. Adicionalmente, Cicua, Méndez y Muñoz (2008), señalan que el consumo de alcohol en nuestro país, desde hace tiempo ha sido visto como una conducta aceptada socialmente, hecho que repercute de manera significativa no solo en el ámbito social y familiar, sino en la salud pública.

Entre tanto, los resultados de este estudio también arrojaron que el $2 \%$ de los participantes presentan un nivel perjudicial, lo que puede conllevar consecuencias para la salud física y mental, así como consecuencias sociales debido a los daños causados por el alcohol; es así que Martínez y Villar (2004), refieren en su investigación un estudio en el que el $31,5 \%$ de los estudiantes del primer año de secundaria reportaron el consumo de alcohol en las últimas dos semanas, y los motivos para su uso varían, yendo desde la necesidad de ser aceptados por un grupo social, conducta aprendida de personas significativas hasta recurso para afrontar conflictos personales. Así mismo, Espada, Griffin, Botvin y Méndez (2003) refieren que algunos de los principales inconvenientes derivados del abuso de alcohol son problemas escolares, afectivos y legales, sexo ocasional, accidentes de tránsito e incluso consumo de otras drogas. En todo caso, por ser el consumo de alcohol una variable subjetiva y socialmente cuestionada, no se tendría claridad de la sinceridad en las respuestas y por tanto de su gravedad.

Por otro lado, para las variables consumo de alcohol e ideación suicida, no encontramos diferencias significativas, lo que coincide con el estudio realizado por Toro, Paniagua, González y Montoya (2009) en la investigación realizada con adolescentes escolarizados de colegios públicos de Medellín. En contraste, Fuentes et al., (2009) encontraron que un porcentaje mucho más elevado de sujetos adolescentes escolarizados de la ciudad de Manizales son dependientes del alcohol (39.2\%), y a su vez este consumo se relaciona con el riesgo suicida. Al analizar la variable consumo de alcohol, es de resaltar que la gran mayoría de la población reportó un consumo moderado y que el $2 \%$ de los individuos participantes reportaron un nivel perjudicial, aspecto que, como es sabido, puede conllevar consecuencias para la salud física y mental, así como consecuencias sociales debido a los daños causados por el mismo (OMS).

A pesar de que los resultados de las relaciones entre estas dos últimas variables no son concluyentes, Evans et al. (2004) encuentran que hay una asociación entre el consumo de alcohol, el intento y la ideación suicida; especialmente con el alto consumo y con el consumo de bebidas con elevado contenido de licor; así mismo, Cardona y Hernández (2007) indican que en efecto el consumo de alcohol aumenta el riesgo suicida ya que el contacto con el organismo modifica sus funciones, y a su vez los estados de conciencia y el pensamiento; mientras que Toro et al. (2009) sugieren que el consumo de alcohol agrava los sentimientos depresivos y disminuye el autocontrol. 
Con respecto a las cifras de consumo de alcohol y los estudios previos sobre la relación entre el consumo y la conducta suicida, proponemos retomar los planteamientos de Martínez y Villar (2004), quienes afirman que es necesario ejercer mayor seguimiento $\mathrm{y}$ vigilancia así como "reforzar el sentimiento de autoeficacia (confianza para rechazar)" y el manejo de la presión social para no caer en el consumo; adicionalmente, y tal como lo plantean Cabra et al., (2010), ya que los adolescentes y las adolescentes se encuentran en una etapa de la vida tan importante en su desarrollo personal, es necesario que se les preste un acompañamiento cercano, identificando la estructura y calidad de sus relaciones familiares, con el fin de establecer estrategias de comunicación efectiva y de resolución de problemas que les permita percibir un buen funcionamiento familiar; ya que como lo mencionamos, éste es un factor de protección para la conducta suicida, teniendo en cuenta que es un período de transición biopsicosocial que se caracteriza por modificaciones corporales y adaptación a nuevas estructuras psicológicas que llevan a la adultez (Silva, 2007).

Si bien los resultados de esta investigación no son concluyentes, proponemos que para futuras investigaciones relacionadas se profundice en el tema del consumo de alcohol, ya que la política Nacional para la reducción del consumo de sustancias psicoactivas y su impacto (Silva, 2007) plantea que el consumo de alcohol es un problema de alta relevancia social, que no solo afecta el desarrollo de la agenda del país sino su capital humano y social, identificando de manera más precisa los factores de riesgo y protección para el consumo en los adolescentes Tunjanos; ya que, como es sabido, Boyacá se encuentra en el tercer lugar por encima de la media nacional de ingesta de alcohol (Estudios Nacionales de Consumo de Sustancias Psicoactivas de Colombia-ENCSPC, 2013); así mismo, porque diferentes autores y autoras han reportado una relación entre el consumo y la ideación suicida (Evans, Hawton, Rodham \& Deeks et al., 2004, Cardona \& Hernández, 2007, Toro et al., 2009, y Fuentes et al., 2009). Al respecto, en Colombia es de resaltar que las políticas públicas pretenden orientar acciones en tres ejes: prevención, mitigación y superación de los daños asociados al consumo de sustancias a través de la promoción de la salud, reduciendo las condiciones de vulnerabilidad de los ciudadanos y ciudadanas (Política Nacional de Reducción del Consumo de Sustancias Psicoactivas-PNRCSP, 2007).

Finalmente, y teniendo en cuenta las cifras de ideación suicida presentadas en este estudio, proponemos la generación de estrategias de prevención e intervención oportuna, tanto a nivel individual como familiar y social, con el fin de garantizar un acompañamiento integral por parte de personal capacitado en el proceso de formación de los individuos adolescentes de la ciudad de Tunja.

\section{Conclusiones}

Teniendo en cuenta que según los resultados encontramos que el $30 \%$ de los sujetos participantes se ubican en un nivel alto de ideación suicida (IS), siendo más alta la proporción en mujeres con respecto a los hombres, sugerimos continuar con el estudio de variables sociodemográficas que permitan mayor claridad en los resultados, con respecto a estudios previos.

Si bien encontramos que el $67 \%$ de las personas participantes refirieron tener un buen funcionamiento familiar, caracterizado por el desarrollo de sus integrantes, y el afrontamiento adecuado de los problemas relacionales y de crisis familiar, es importante profundizar sobre elementos tales como la influencia de los pares y del contexto social en general, como elementos de riesgo y/o protección en el fortalecimiento de las redes familiares.

Según la alta prevalencia de ideación suicida identificada en este estudio y su asociación con la disfuncionalidad familiar, proponemos continuar con otros estudios relacionados que permitan profundizar en aspectos asociados a la dinámica familiar, de tal manera que se pueda hacer un aporte en la generación de políticas públicas en pro del mejoramiento de la calidad de vida de las personas adolescentes y de sus familias.

Debido a que el presente estudio tuvo como limitante el número de variables 
analizadas, sugerimos seguir profundizando en otros factores que puedan estar asociados a la ideación suicida, con el fin de generar o fortalecer los modelos explicativos de dicho fenómeno y avanzar en el proceso de generación de programas de prevención del riesgo y promoción de los factores protectores de la salud mental de los adolescentes y las adolescentes.

Si bien identificamos en la mayoría de los sujetos participantes un consumo moderado de alcohol, lo cual es de resaltar teniendo en cuenta su edad, esta variable no se asocia con la ideación suicida en dicha población, como se presenta en otros grupos etarios.

\section{Lista de referencias}

Bailador, P., Viscardi, N. \& Dajas, F. (1997). Desesperanza, conducta suicida y consumo de alcohol y drogas en adolescentes de Montevideo. Revista Médica de Uruguay, 13 (3), pp. 213-223.

Cabra, O., Infante, D. \& Sossa, F. (2010). El suicidio y los factores de riesgo asociados en niños y adolescentes. Revista Médica Sanitas 13 (2), pp. 28-35.

Camacho, I. (2005). Factores psicosociales relacionados con el consumo de sustancias psicoactivas en estudiantes de secundaria. Cuadernos Hispanoamericanos de psicología, 5 (1), pp. 41-56.

Campo, G., Roa, J., Pérez, A., Salazar, O., Piragauta, C., López, L. \& Ramírez, C. (2003). Intento de suicidio en niños menores de 14 años atendidos en el Hospital Universitario del Valle, Cali. Colombia Medica, 34 (1), pp. 9-16.

Cardona, A. \& Hernández, H. (2007). Consumo de alcohol y otros psicoactivos en estudiantes universitarios y su relación con aspectos de la calidad de vida y factores psicosociales. Tesis de pregrado. Universidad de Antioquia, Medellín, Colombia.

Carvajal, G. \& Caro, C. (2011). Ideación suicida en la adolescencia: Una explicación desde tres de sus variables asociadas en Bogotá, 2009. Revista Colombia Médica, 42 (2) (supl. 1), pp. 45-56.
Ceballos, G., Suarez, Y., Suescún, J., Gamarra, M., González, K. \& Sotelo, A. (2015). Ideación suicida, depresión y autoestima en adolescentes escolares de Santa Marta. Revista Duazary, 12 (1), pp. 15-22.

Cicua, D., Meléndez, M. \& Muñoz, L. (2008). Factores en el consumo de alcohol en adolescentes. Pensamiento psicológico, 4 (11), pp. 115-134.

Córdova, M., Rosales, J., Guzmán, B. \& Zúñiga, G. (2013). Características del estudiante con y sin ideación suicida del colegio de bachilleres de san Luis Potosí (México). Revista Salud y Sociedad, 4 (2), pp. 120134.

De Wilde, E. (2000). Adolescent suicidal behaviour: A general population perspective. En K. Hawton \& K. van Heeringen (eds.) The international handbook of suicide and attempted suicide, (pp. 249-259). Chichester: John Wiley and Sons.

Espada, J., Griffin, K., Botvin \& Méndez, X. (2003). Adolescencia: Consumo de alcohol y otras drogas. Papeles del psicólogo, 23 (84), pp. 9-17.

Evans, E., Hawton, K. \& Rodham, K. (2004). Factors associated with suicidal phenomena in adolescents: A systematic review of population-based studies. Clinical Psychology Review, 24 (8), pp. 957-979.

Evans, E., Hawton, K., Rodham, K \& Deeks, J. (2004). The prevalence of suicidal phenomena in adolescents: A systematic review of population-based studies. Suicide life threat Behavior, 35 (3), pp. 239-250.

Forero, L., Avendaño, M., Duarte, Z. \& Campo, A. (2006). Consistencia interna y análisis de factores de la escala Apgar para evaluar el funcionamiento familiar en estudiantes de básica secundaria. Revista Colombiana de psiquiatría, 35 (1), pp. 23-29.

Fuentes, M., González, A., Castaño, J., Hurtado, C., Ocampo, P., Páez, M. (...) Zuluaga, L. (2009). Riesgo suicida y factores relacionados en estudiantes de $6^{\circ}$ a $11^{\circ}$ grado en colegios de la ciudad de Manizales (Colombia). 2007-2008. Archivos de Medicina, 9 (2), pp. 110-122. 
García, M. \& Calíope, C. (1998). Incidence and predictors of suicidal behaviors in a longitudinal sample of young adolescents. Departamento de Medicina Legal. Facultad de Medicina Universidad de la República, 37 (6), pp. 612-619.

Gómez, C., Rodríguez, M., Bohórquez, A., Diazgranados, N., Ospina, M. \& Fernández, C. (2002). Factores asociados al intento de suicidio en la población colombiana. Revista Colombina de Psiquiatría, 31 (4), pp. 283-298.

Hernández, R., Fernández, C. \& Baptista, P. (2000). Metodología de la investigación. México, D. F.: McGrawHill.

Instituto Nacional de Medicina Legal y Ciencias Forenses-INMLCF (2013). Forensis 2013. Datos para la vida. Bogotá, D. C.: Panamericana.

Martínez, G. \& Villar, M. (2004). Estudio descriptivo del uso de drogas en adolescentes de educación media superior de la ciudad de Monterrey, Nueva León, México. Revista Latinoamericana de Enfermería, 12, pp. 391-397.

Ministerio de Justicia de Colombia, Ministerio de Salud de Colombia \& Observatorio de Drogas de Colombia (2013). Estudio nacional de consumo de sustancias psicoactivas en Colombia (ENCSPE). Recuperado de:

http://www.unodc.org/documents/ colombia/2014/Julio/Estudio_de Consumo UNODC.pdf

Ministerio de Protección Social de ColombiaMPS (2005). Encuesta sustancias psicoactivas. Recuperado de: http://www. minsalud.gov.co/paginas/default.aspx

Organización Mundial de la Salud-OMS (2001). Cuestionario de Identificación de los Trastornos debidos al Consumo de Alcohol. Recuperado de:

http://www.who.int/substance abuse/ activities/en/AUDITmanualSpanish.pdf

Organización Mundial de la Salud-OMS (2014). Global status report on alcohol and healt. Recuperado de:

http://blogs.20minutos.es/el-nutricionistade-la-general/2014/05/19/informe-oms- 2014-consumo-de-alcohol-y-salud-en-elmundo/

Organización Mundial de la Salud-OMS (2015). Prevención del suicidio. Factores de riesgo. Recuperado de: http://www. who.int/mental health/prevention/es/

Osman, A., Gutiérrez, P., Jiandani, J., Barrios, F., Linden, S. \& Truelove, R. (2003). A preliminary validation of the positive and negative suicide ideation (Pansi) Inventory with normal adolescent samples. Journal of Clinical Psychology, 59, pp. 493-512.

Paniagua, R., González, C. \& Rueda, S. (2014). Orientación al suicidio en adolescentes en una zona de Medellín, Colombia. Revista Facultad Nacional de Salud Pública, 32 (3), pp. 314-321.

Pérez, B., Rivera, L., Atienzo, E., de Castro, F., Leyva, A. \& Chávez, R. (2010). Prevalencia y factores asociados a la ideación e intento suicida en adolescentes de educación media superior de la República mexicana. Salud pública de México, 52 (4), pp. 324-333.

Pérez, I., Téllez, D., Vélez, A. \& Ibáñez, M. (2012). Caracterización de factores asociados con comportamiento suicida en adolescentes estudiantes de octavo grado, en tres colegios bogotanos. Revista Colombiana de Psiquiatría, 41 (1), pp. 2647.

Pérez, M., Vianchá, M., Martínez, L. \& Salas, I. (2014). El maltrato familiar y su relación con la ideación suicida en adolescentes escolarizados de instituciones públicas y privadas de las ciudades de Tunja, Duitama y Sogamoso. Psicogente, 17 (31), pp. 8092.

Ramírez, M. \& Andrade, D. (2005). La familia y los factores de riesgo relacionados con el consumo de alcohol y tabaco en los niños y adolescentes (Guayaquil, Ecuador). Revista Latino-americana Enfermagem, 13 (número especial), pp. 813-818.

Sánchez, J., Villarreal, M., Musitu, G. \& Martínez, B. (2010). Ideación suicida en adolescentes: Un análisis psicosocial. Intervención psicosocial, 19 (3), pp. 279287. Doi:10.5093/in2010v19n3a8.

Saunders, J., Aasland, O., Babor, T., De La Fuente J. \& Grant, M. (1993). Development 
of the Alcohol Use Disorders Identification Test (Audit): WHO collaborative project on early detection of persons with harmful alcohol consumption-II. Addiction, $88, p p$. 791-804.

Silva, I. (2007). La adolescencia y su interrelación con el entorno. Madrid: Ministerio de sanidad, Servicios Sociales e Igualdad. Recuperado de: http://www. injuve.es/observatorio/familia-pareja-eigualdad-de-genero/la-adolescencia-y-suinterrelacion-con-el-entorno.

Smilkstein, G. (1978). The family Apgar. A proposal for a family function test and its used for physicians. The Journal of Family Practice, 6 (6), pp. 1231-1239.

Suárez, M. \& Alcalá, M. (2014). Apgar familiar: Una herramienta para detectar disfunción familiar. Revista Médica La Paz, 20 (1), pp. 53-57.

Toro, D., Paniagua, R., González, C. \& Montoya, B. (2009). Caracterización de adolescentes escolarizados con riesgo de suicidio, Medellín, 2006. Revista Facultad Nacional de Salud Pública, 27 (3), pp. 302-308.

Valencia, L. (2014). Factores de riesgo $y$ protección de ideación suicida en adolescentes de una institución educativa de Chiquinquirá. Tesis de pregrado inédita. Universidad Pedagógica y Tecnológica de Colombia, Bogotá, Colombia.

Vargas, H. \& Saavedra, J. (2012). Factores asociados a la conducta suicida en adolescentes. Revista de Neuropsiquiatría, 75 (1), pp. 19-28.

Villalobos-Galvis, F. (2009a). Ideación suicida en jóvenes: Formulación y validación del modelo integrador explicativo en estudiantes de secundaria y superior. Tesis doctoral inédita. Universidad de Granada, España. Recuperado de:

http://hera.ugr.es/tesisugr/1805982x.pdf

Villalobos-Galvis, F. (2009b). Situación de la conducta suicida en estudiantes de colegios y universidades de San Juan de Pasto, Colombia. Salud Mental, 32 (2), pp. $165-$ 171. 